\title{
Sextant
}

Revue de recherche interdisciplinaire sur le genre et la sexualité

36 | 2019

No children, no cry

\section{Le choix de la non-maternité dans l'Antiquité tardive}

Marine Tesson

(2) OpenEdition

Journals

Édition électronique

URL : https://journals.openedition.org/sextant/391

DOI : $10.4000 /$ sextant.391

ISSN : 2795-8736

Éditeur

Éditions de l'Université de Bruxelles

Édition imprimée

Date de publication : 1 décembre 2019

Pagination : 115-134

ISBN : 978-2-8004-1705-9

ISSN : $1370-267 X$

Référence électronique

Marine Tesson, « Le choix de la non-maternité dans l'Antiquité tardive », Sextant [En ligne], 36 | 2019, mis en ligne le 01 novembre 2021, consulté le 08 décembre 2021. URL : http://

journals.openedition.org/sextant/391 ; DOI : https://doi.org/10.4000/sextant.391

\section{(ब) $\odot \odot$}

La revue Sextant est mise à disposition selon les termes de la Licence Creative Commons Attribution Pas d'Utilisation Commerciale - Partage dans les Mêmes Conditions 4.0 International. 


\title{
Le choix de la non-maternité dans l'Antiquité tardive
}

\author{
Marine TESSON
}

\begin{abstract}
Résumé :
L'Antiquité tardive est une période où des femmes ont pu faire le choix d'une nonmaternité volontaire. En effet, dans les premiers siècles de l'Empire, si les femmes avaient une possibilité de contrôler le nombre d'enfants à qui elles donnaient le jour, bien qu'elles dussent avoir pour cela l'accord de leur mari, le rôle des femmes était d'abord d'être mère. Le christianisme, qui prend son essor au III siècle et devient une religion officielle au IV siècle, propose de nouvelles normes à la société romaine. En effet, son souci n'est plus la perpétuation des lignées, mais le salut individuel après la mort. Tout un mouvement ascétique va encourager les femmes à renoncer au mariage au profit d'une vie de virginité et de prière, ou si elles sont mariées, à envisager la continence. En parallèle, l'avortement est de plus en plus désapprouvé socialement. La législation va être modifiée par l'empereur Constantin : désormais, l'état de célibat n'est plus pénalisé. Ce choix de non-maternité, dans le couple ou hors du couple, s'accompagne donc d'un choix de non-sexualité. Pour autant transparaît dans les sources antiques l'existence de femmes qui, tout en profitant de cette latitude de choix de non-maternité, ne s'inscrivent pourtant pas dans une perspective d'obéissance aux normes religieuses d'abandon de leur sexualité. Elles profitent de cet espace interstitiel qui leur est octroyé pour vivre une vie de femme sans maternité.
\end{abstract}

\begin{abstract}
:
During Late Antiquity, women could take for the first time the decision of the nonmaternity. Indeed, through the first centuries of the Roman Empire, if women could control how many children they had, the duty of a woman was first to give birth. The Christianism, which rose in the third century and became the official religion of the Empire in the fourth century, offers new standards to the Roman society. The Christian preoccupation is now the afterlife and not ensures that lineages are prolonged. An ascetic movement encourages women to renounce to marriage. Simultaneously, the abortion meets more and more disapproval in society. The law changes with the Emperor Constantine: now, to be celibate is no more penalized. This choice of nonmaternity, in the couple or outside the couple, has to be supported by the absence of sexuality. Nevertheless, according to our sources, there are lots of women in Antiquity who make profits of the new standards, without obey to religious recommendations and live their lives without childbirth in this interstitial space.
\end{abstract}


Quand Salomé déclare : «J'ai donc bien fait de ne pas enfanter », comme s'il ne convenait pas d'assumer la procréation, le Seigneur répondit ainsi : « Mange de toute plante, mais de celle qui est amère ne mange pas ${ }^{1}$.

Cet extrait de l'évangile apocryphe des Égyptiens, évangile non reconnu par l'Église, est très utilisé dans les communautés chrétiennes des premiers siècles de notre ère. Les chrétiens gnostiques, qui défendent l'idée que les êtres humains sont des âmes divines emprisonnées dans un monde matériel ${ }^{2}$, ont vu dans ce passage l'assentiment du Christ au refus de Salomé d'enfanter. D'autres chrétiens l'ont plutôt interprété comme une remarque sur l'inutilité de regretter ce qui est, et non comme un regret d'être mère. Pourtant Luc affirme :

«Jésus se tourna vers elles, et dit : Filles de Jérusalem, ne pleurez pas sur moi; mais pleurez sur vous et sur vos enfants. Car voici, des jours viendront où l'on dira : heureuses les stériles, heureuses les entrailles qui n'ont point enfanté, et les mamelles qui n'ont point allaité !» ${ }^{3}$. Dès les premiers textes chrétiens, le refus de la procréation apparaît donc envisageable.

Le thème de la non-maternité a rarement été étudié pour lui-même en histoire ancienne, mais de nombreux travaux y font référence. Les plus pertinents sont les recherches sur la contraception et l'avortement, menées dès 1936 par N. Himes, suivi par K. Hopkins, J. T. Noonan, E. Eyber, S. Gourevitch, A. Rousselle, A. McLaren ou J. M. Riddle, ce dernier ayant étudié l'effet médical réel des remèdes conseillés par les médecins antiques ${ }^{4}$ mais dont les conclusions sont discutées par B. W. Frier sur leur usage concret dans la société romaine ${ }^{5}$. L'abandon des enfants et l'infanticide ont été amplement traités ${ }^{6}$. Les nombreuses études sur l'ascétisme ont aussi abordé

${ }^{1}$ Clément D’Alexandrie, Stromates, III, 9, 66, 2 citant l'évangile grec des Égyptiens, qui ne nous est pas parvenu autrement que par des fragments, in Écrits apocryphes chrétiens, Paris, Gallimard (coll. « La Pléiade »), 1997, p. 477.

2 P. Наdot, «Le Gnosticisme», in Dictionnaire de l'Histoire du christianisme, Paris, Encyclopaedia Universalis, Albin Michel, 2014, p. 641.

${ }^{3}$ Luc, 23, 28-29.

${ }^{4}$ N. Himes, Medical history of contraception, Baltimore, The Williams and Wilkins Company, 1936 ; K. Hopkins, "Contraception in the Roman Empire », Comparative Studies in Society and History, vol. 8(1), 1965, p. 124-151 ; J. T. Noonan, Contraception et mariage, Paris, Le Cerf, 1969 [1966] ; E. EyBER, « Family planning in Graeco-Roman Antiquity », Ancient Society, vol 11/12, Louvain, 1980-1981, p. 5-82 ; S. Gourevitch, Le mal d'être femme. La femme et la médecine dans la Rome antique, Paris, Les Belles Lettres, 1984 ; A. Rousselle, Porneia, Paris, Presses universitaires de France, 1983 ; A. McLaren, A History of contraceptions from Antiquity to the Present day, Oxford, Blackwell, 1990 ; J. M. RiddLe, Contraception and Abortion from the Ancient world to Renaissance, Cambridge (Mass.), Harvard University Press, 1992.

${ }^{5}$ B. W. FrIER, « Natural Fertility and Family Limitation in Roman Marriage », Classical Philology, vol. 89, n 4, 1994, p. 318-333.

6 J. E. Boswell, « Expositio and Oblatio: The Abandonment of Children and the Ancient and Medieval Family », The American Historical Review, vol. 89, $\mathrm{n}^{\circ}$ 1, 1984, p. 10-33; J. E. Boswell, The Kindness of Strangers. The Abandonment of Children in Western Europe from Late Antiquity to the Renaissance, New York, Pantheon books, 1988 ; P. GARNSEY, « Child 
à la marge cette question de la non-maternité, avec l'œuvre incontournable de P. Brown ${ }^{7}$ mais plus récemment des publications comme celle de V. Vuolanto ${ }^{8}$. La maternité elle-même est l'objet d'études récentes ${ }^{9}$. Les trois dernières décennies ont globalement abouti au renforcement des recherches sur les femmes dans l'Antiquité, avec une volonté de les replacer au cœur des études historiques ${ }^{10}$. En revanche, si ces thématiques sont de mieux en mieux connues et étudiées, le biais des sources conservées, essentiellement écrites par des auteurs masculins, continue de constituer un obstacle à l'étude du point de vue féminin. Un intérêt majeur de ce numéro de Sextant est alors de nous amener à adopter le regard des femmes sur la question de la non-maternité, en interrogeant leur latitude de choix. À ce propos, l'Antiquité tardive constitue une période charnière. Yvonne Knibiehler ${ }^{11}$ met ainsi en évidence la rupture que constitue l'Antiquité tardive dans l'assimilation de la femme à la mère. La loi de l'empereur Constantin de $320{ }^{12}$ marque un tournant radical : le célibat n'est plus légalement pénalisé, de même que l'absence d'enfants. Il est donc possible de s'interroger sur le choix féminin d'une non-maternité volontaire, entre le $\mathrm{III}^{\mathrm{e}}$ et le $\mathrm{VI}^{\mathrm{e}}$ siècle de notre ère, dans l'Empire romain et les royaumes romano-barbares qui revendiquent son héritage.

En premier lieu, j'étudierai quelles sont les possibilités de choix de non-maternité pour les femmes. L'intervention du christianisme dans ce débat offre de nouvelles possibilités aux femmes : elles peuvent choisir de ne pas avoir d'enfants. Mais elles doivent dans le même temps renoncer à toute sexualité, ce que j'étudierai dans un deuxième temps. Pourtant, nous verrons dans une troisième partie que sans obéir à cette contrainte de la chasteté, des femmes font le choix de refuser la maternité.

\section{La non-maternité dans la société romaine : quel choix pour les femmes?}

Au début de notre ère, l'empereur Auguste met en place une législation qui pénalise les célibataires. Il restreint leurs droits en matière d'héritage, tandis que les

rearing in Ancient Italy », in D. I. Kertzer et R. P. SAller (eds.), The Family in Ancient Italy from Antiquity to the Present, New Haven, Londres, Yale University Press, 1992 ; W. V. HarRIs, " Child-Exposure in the Roman Empire », Journal of Roman Studies, 84, p. 1-22 ; M. CorbiER, " Child Exposure and Abandonment », in S. Dixon (ed.), Childhood, Class and Kin in the Roman World, Londres, Routledge, 2001, p. 52-73.

7 P. Brown, Le Renoncement à la chair, Paris, Gallimard, 1996 [1995].

${ }^{8}$ V. Vuolanto, Children and Asceticism in Late Antiquity, Burlington, Farnham, Ashgate, 2015.

9 C. Leyser et L. Smith, Motherhood, Religion and Society in Medieval Europe, 4001400, Burlington, Farnham, Ashgate, 2011.

${ }^{10}$ G. Duby et M. Perrot (dir.), Histoire des femmes en Occident, Paris, Plon, 1990-1991 (5 volumes) ou encore J. M. Bennett et R. Mazo Karras (eds.), The Oxford handbook of women and gender in Medieval Europe, Oxford, Oxford University Press, 2013 pour ne citer que quelques exemples.

${ }^{11}$ Y. KniBIenler, Histoire des mères et de la maternité en Occident, Paris, Presses universitaires de France, 2000.

12 Code Théodosien VIII, 16, 1, version latine et traduction française, in P. LAURENCE, Les droits de la femme au Bas-Empire romain : le Code Théodosien, Neuville-sur-Saône, Chemins de traverse, 2012. 
citoyennes mères de trois enfants et les affranchies mères de quatre enfants échappent à la tutelle qui pèse sur les femmes et obtiennent des droits juridiques élargis ${ }^{13}$. Pourtant cette législation qui se veut très favorable à la maternité se heurte à la réalité des pratiques. Contraception, avortement et abandon à la naissance sont fréquents dans la société romaine ${ }^{14}$, sans que cette dernière hiérarchise ces différentes pratiques ${ }^{15}$, même si elles sont bien différenciées dans l'esprit des Romains ${ }^{16}$. Nulle dimension morale n'est alors invoquée. Les Romains se placent du côté de la finalité plutôt que de celui des moyens employés. Quelle place peuvent alors tenir les femmes dans le refus de la maternité?

Les méthodes employées ont été longuement décrites par divers auteurs, dont Noonan, Gourevitch et plus récemment Riddle ${ }^{17}$. Je vais surtout m'intéresser à l'efficacité et à l'accessibilité de ces méthodes pour les femmes, car le choix de nonmaternité est conditionné à leur possibilité d'action. La classe sociale joue-t-elle un rôle ? Prolongeant une logique déjà présente chez Juvénal au $\mathrm{II}^{\mathrm{e}}$ siècle ${ }^{18}$, Ambroise de Milan, au IV ${ }^{\mathrm{e}}$ siècle, l'affirme. Il différencie les femmes pauvres, qui ne peuvent pas mettre fin à leur grossesse, des femmes riches qui « refusent leur propre fœtus en leur sein et, par des potions, détruisent les promesses de leur matrice dans leurs entrailles $\gg{ }^{19}$. Les médecins proposent des recettes de potions abortives. Soranos d'Éphèse évoque au début du $\mathrm{II}^{\mathrm{e}}$ siècle un mélange de graines de giroflée jaune, de myrte, de myrrhe et de poivre blanc à prendre dans du vin pendant trois jours ${ }^{20}$, ingrédients exotiques et coûteux. L'accès à de telles recettes comme aux ingrédients ne pouvait être que l'apanage de femmes riches. Mais les vertus de certaines plantes pouvaient correspondre à un savoir populaire. Ovide en témoigne dans ses Héroïdes où il raconte les mésaventures de la fille d'Éole qui, enceinte, reçoit l'aide de sa nourrice pour tenter d'avorter : "Que d'herbes, que de médecines ma nourrice ne m'apporta-t-elle pas, et de sa main audacieuse, ne fit-elle pas pénétrer en moi» ${ }^{21}$. À côté des médecins cultivés des hautes classes sociales, des guérisseurs devaient aussi

13 A. Berger, « Encyclopedic Dictionary of Roman Law », Transactions of the American Philosophical Society, n 43.2, 1953, p. 530.

${ }^{14}$ Minucius Felix, dans son Octavius daté de la $1^{\text {ère }}$ moitié du $\mathrm{III}^{\mathrm{e}} \mathrm{s}$. parle tant de l'usage de drogue que de la pratique de l'exposition ou encore de l'étouffement ou de l'étranglement pour se débarrasser des enfants non désirés. Il les présente évidemment comme des pratiques païennes, mais elles ont sans doute une large diffusion dans la société. Octavius, 30, 2, Paris, Les Belles Lettres, 1964.

${ }^{15}$ B. Rawson, Children and Childhood in Roman Italy, Oxford, Oxford University Press, 2003, p. 114-115. http://books.openedition.org/pucl/784?lang=fr, paragraphe 38.

16 J. M. RidDLE, op. cit., p. 24-25.

${ }^{17}$ S. Gourevitch, op. cit. ; et J. T. Noonan, op. cit., mais aussi J. M. Riddle, op.cit. ; $c f$. note 4 pour plus de références.

18 Juvénal, Satires, VI, 592-600, Paris, Les Belles Lettres, 1951. Il met en évidence l'usage de poisons par les femmes riches pour mettre fin à leur grossesse.

19 Ambroise de Milan, Hexaméron, 5, 18, 58; Corpus scriptorum ecclesiasticorum latinorum (CSEL), t. 32, 184 et J. T. NoONAN, op. cit., p. 132.

${ }^{20}$ Soranos D'Éphèse, Maladies des femmes, 1, 20, 80-90, t. 1, Paris, Les Belles Lettres, 1988.

${ }^{21}$ Ovide, Hérö̈des, XI, Canacé à Macarée, Paris, Les Belles Lettres, 1928, p. 66. 
intervenir, la nourrice pouvant elle-même connaître les herbes ou faire appel à une spécialiste. Les potions recommandées par les médecins pouvaient être efficaces ${ }^{22}$, mais la diffusion des recettes pouvait sans doute poser problème et aboutir à des naissances non-désirées.

L'idéal restait de ne pas tomber enceinte. Pour A. Rousselle, les matrones, après avoir donné à leur époux les héritiers attendus, devenaient continentes afin d'éviter les dangers de nouvelles grossesses, tandis que leurs maris se tournaient vers les concubines, les esclaves et les courtisanes pour vivre leur sexualité ${ }^{23}$. Même si cette hypothèse peut être valable dans certains cas, il est probable que le désir du mari pour son épouse ne pouvait être freiné par de telles considérations, et elle ne résout pas le problème pour la majorité des femmes. Le recours à des méthodes contraceptives devait donc exister, même si la littérature latine est discrète à ce sujet. J. T. Noonan indique que la littérature juive témoigne de l'usage fréquent de pessaires, dispositif généralement en tissu ou en laine, parfois en fibres de plantes recouvertes de résine que l'on introduit dans le vagin. Il considère que cette méthode de contraception devait être diffusée dans l'Empire romain, car très facile à utiliser ${ }^{24}$. Dans cette perspective, la mention de bandages pour avorter par Hippolyte de Rome au $\mathrm{II}^{\mathrm{e}}$ siècle témoigne peutêtre de leur usage en matière de contraception ${ }^{25}$. Gourevitch doute de leur efficacité ${ }^{26}$, et Riddle conclut que certains sont efficaces avec des taux d'efficacité de $60 \%$ à $70 \%$ selon la composition ${ }^{27}$, ce qui laisse une large marge à la maternité non-désirée. Le coitus interruptus devait aussi être employé même s'il est difficile d'en connaître les proportions ${ }^{28}$. Son absence de mention dans les sources latines se justifie car il rendrait inutiles les prescriptions savantes des médecins ${ }^{29}$. De plus, l'idée que l'homme doive interrompre son plaisir sexuel était sans doute inacceptable pour l'esprit masculin romain. L'idée d'une période de stérilité féminine avant et pendant les règles est aussi commune $^{30}$. Lucrèce, au I ${ }^{\text {er }}$ siècle, considère que les «déhanchements lascifs» de la courtisane lui permettent de ne pas tomber enceinte. Les relations sexuelles infertiles existaient et l'homosexualité féminine était une forme de sexualité permettant d'éviter la maternité ${ }^{31}$. Le recours au surnaturel était aussi fréquent : des amulettes devaient

${ }_{22}$ J. M. RidDLE, dans Contraception and Abortion from the Ancient world to Renaissance, op. cit., donne ainsi l'exemple de la peau ou de l'écorce de grenade prescrite par Soranos d'Ephèse qui semble fonctionner efficacement, testée sur deux groupes différents de cobayes, en laboratoire (p. 25-26). Il conclut que 8 des 10 recettes de Soranos pouvaient fonctionner en tant que contraceptifs et/ou abortifs (p. 30).

${ }^{23}$ A. Rousselle, op. cit., p. 344.

24 J. T. NoOnan, op. cit., p. 19 et 30.

${ }^{25}$ Hippolyte de Rome, Philosophumena, Paris, Rieder, 1928, IX, 2, pour la version française. P. CRUICE, Paris, 1860, pour la version grecque et sa traduction en latin, IX, 11.

${ }^{26}$ S. GOUREVITCH, op. cit., p. 198-199.

27 J. M. RidDLE, op. cit., p. 35-37.

${ }^{28}$ Pour une discussion sur cette question, $c f$. J. M. RidDLE, op. cit.

29 J. T. NoOnan, op. cit., p. 27.

30 Soranos d'ÉphÈse, Maladies des femmes, 1, 20, 36, op. cit.

31 E. BRooten, Love between women, early Christian responses to female homoeroticism, Chicago, University of Chicago Press, 1996 ; S. BoeHringer, L'Homosexualité féminine dans l'Antiquité grecque et romaine, Paris, Les Belles Lettres, 2007. 
permettre d'éviter la maternité, et sont recommandées jusqu'au $\mathrm{VI}^{\mathrm{e}}$ siècle par le médecin chrétien de la cour de Justinien Aëtios d'Amida, en complément de différents moyens de contraception et d'avortement qu'il présente sans réserves morales ${ }^{32}$. La plupart des médecins de l'Antiquité tardive ayant tout aussi peu de réserves ${ }^{33}$.

Toutes ces méthodes laissaient à désirer, à cause des problèmes de diffusion à travers les différentes couches de la société, mais aussi par méconnaissance de ce qui fonctionnait effectivement et ce qui était au contraire complètement inutile bien que recommandé ${ }^{34}$. L'échec de la nourrice dans les Hérö̈des en est l'illustration : «Hélas! Trop vivace, l'enfant résista aux manœuvres ${ }^{35}$. Les risques encourus par les femmes qui utilisent des méthodes contraceptives et abortives sont soulignés par les médecins comme par les témoins du temps ${ }^{36}$. Pour Frier, les méthodes de limitation des naissances n'ont pas de réel impact à l'échelle de l'ensemble de la population romaine et ne sont pas pratiquées par la majorité ${ }^{37}$. L'abandon de l'enfant à la naissance reste la méthode la plus sûre. Si l'enfant n'est pas reconnu par son père, il n'a pas d'existence sociale et est exposé dans la rue ${ }^{38}$. Pour Sénèque le Rhéteur, au $\mathrm{I}^{\mathrm{er}}$ siècle, l'exposition des enfants sert même à corriger les caprices du sort qui a fait les uns pauvres et les autres riches à travers l'adoption ${ }^{39}$. Les motivations des plus riches à l'abandon, notamment éviter la division d'un patrimoine, ou l'illégitimité soupçonnée, sont différentes de celles des pauvres, pour qui l'exposition permet de résoudre l'insoluble problème d'une bouche supplémentaire à nourrir. Cette pratique était amplement diffusée ${ }^{40}$. Les filles sont plus souvent concernées que les garçons même si la disproportion entre les sexes est difficilement mesurable, les infirmités pouvant toucher sans distinction les deux sexes par exemple ${ }^{41}$. La pratique continue d'exister dans l'Antiquité tardive ${ }^{42}$.

Mais dans la société romaine, ce choix n'est pas féminin. Le dispositif légal de la puissance paternelle qui met la femme sous la tutelle de son père puis de son mari limite d'autant sa liberté de choix ${ }^{43}$. Plutarque, lorsqu'il raconte la vie de Romulus, fondateur de Rome, lui prête la loi suivante : un mari peut répudier sa femme pour avoir

32 Aëtios D’Amida, Médecine, 16, 17, Leipzig, S. Zervos, 1901.

${ }_{33}$ J. M. RIDDLE, op. cit., p. 90-101.

${ }^{34}$ K. Hopkins, op. cit., p. 150.

${ }^{35}$ Ovide, Hérö̈des, XI, Canacé à Macarée, op. cit., p. 66.

${ }^{36}$ Y. KNiBIEhler, op. cit., p. 17, donnant l'exemple de Soranos d'Ephèse ou encore E. Eyber, op. cit., p. 51-52, différentes références.

${ }^{37}$ B. W. FrIER, op. cit., particulièrement p. 318-319 et 327-333 pour sa discussion sur les conclusions de J. M. RIDDLE.

38 P. VeYne, «L'Empire romain», in G. Duby et P. Ariès (dir.), Histoire de la vie privée, t. 1, Paris, Le Seuil, 1985, p. 23-26.

39 SÉNÈQue le RHÉTEUR, Controverses et suasoires, introduction, traduction et notes d'H. BornecQue, Paris, Garnier, 1932.

${ }^{40}$ Voir références en note 5.

${ }^{41}$ W. V. Harris, loc. cit., p. 11.

42 J. C. TATE, "Christianity and the Legal Status of Abandoned Children in the Later Roman Empire », Journal of Law and Religion, vol. 24, n 1, 2008/2009, p. 123-141.

${ }^{43}$ Y. KNIBIEHLER, op. cit., p. 13-14. 
tué son enfant à l'aide de pharmakeia ${ }^{44}$. Ce terme peut tout aussi bien faire allusion au poison qu'à une potion abortive. Si la législation du roi de Rome au VIII ${ }^{\mathrm{e}}$ siècle avant notre ère est d'une historicité douteuse, elle reflète toutefois le mode de pensée des Romains. Le choix de ne pas avoir d'enfant est un choix masculin. Si c'est la femme qui fait ce choix, même après la mort de son mari, ou après un divorce, elle doit être punie car l'enfant appartient au père. Dans un de ses plaidoyers, Cicéron rapporte un jugement auquel il a assisté, en 79 avant notre ère : «Je me souviens que pendant mon séjour en Asie une femme de Milet, pour avoir reçu des héritiers subrogés ${ }^{45}$ une somme d'argent et s'être fait avorter en conséquence par des produits médicaux, fut condamnée de la peine capitale : rien n'était plus juste, puisqu'elle avait anéanti les espoirs d'un père, le souvenir d'un nom, le soutien d'une race, l'héritier d'une famille, un citoyen destiné à la République. ${ }^{46}$ Cette femme s'oppose à la volonté de son mari, alors que la toute-puissance de ce dernier sur leurs enfants prévaut au-delà de son décès ${ }^{47}$. Elle ne mérite donc que la mort. La législation à ce sujet remonterait selon Ulpien (juriste sous Septime Sévère et Caracalla) à Marc-Aurèle et Lucius Verus, co-empereurs entre 161 et 169. Elle mettait en place l'obligation d'une femme divorcée à donner naissance à l'enfant de son ancien mari si elle est enceinte ${ }^{48}$. Si sa rédaction sous Marc-Aurèle n'est pas prouvée, son long développement par Ulpien montre que c'est un objet de préoccupation à la fin du $\mathrm{II}^{\mathrm{e}}$ siècle. Ulpien est encore mentionné plus loin dans le Digeste pour la condamnation au bannissement d'une femme qui avorte ${ }^{49}$. Une citation de Tryphonius mentionne ensuite l'anecdote de Cicéron et un rescrit des empereurs punissant l'avortement dans ce type de cas par un exil temporaire ${ }^{50}$. L'identité des empereurs peut être précisée grâce à une autre loi : ce sont Septime-Sévère et Caracalla. Le rescrit est donc émis entre 198 (où Caracalla est associé au trône) et 211 (mort de Septime Sévère) et condamne bien à l'exil temporaire les femmes ayant avorté sans le consentement de leur ex-mari ${ }^{51}$.

Pourtant, les femmes prenaient des contraceptifs et avortaient sans forcément l'accord de leur mari. Juvénal raille en 115 les riches patriciennes : " sur un lit doré, on ne voit guère de femmes en couches, tant sont efficaces les pratiques et les drogues qui rendent les femmes stériles et pour un prix fixé d'avance, tuent les enfants dans le sein de leur mère. Réjouis-toi, pauvre homme, présente toi-même la potion, quelle

${ }_{44}$ Plutarque, Vie de Romulus, 22, 3, Vies, t. 1, Paris, Les Belles Lettres, 1957, p. 89-90.

${ }^{45}$ C'est-à-dire l'héritier second, heres secundus, celui qui hérite dans le cas où le premier héritier, ici l'enfant, vient à mourir. Cicéron, Discours, Tome VIII, Pour Cluentius, 11, 32, note 2, Paris, Les Belles Lettres, 1953, p. 78.

${ }^{46}$ CicÉron, op. cit. 11, 32, p. 78.

47 Le code théodosien oblige toujours la famille d'un défunt à suivre les prescriptions que celui-ci avait faites avant sa mort quant au mariage futur de ses enfants. Code Théodosien III, 5, 12, op. cit.

${ }^{48}$ Digeste, 25, 4, in D. Gaurier (éd.), Les 50 livres du Digeste de l'Empereur Justinien : comprenant pour la première fois en français La Palingénésie du commentaire sur l'édit du préteur d'Ulpien (d'après Otto Lenel), Nantes, Mémoires du droit, 2017.

49 Digeste, 48, 8, 8, op. cit.

${ }^{50}$ Digeste, 48, 19, 39, op. cit.

${ }^{51}$ Digeste, 47, 11, 4, op. cit. 
qu'elle soit, car si elle voulait bien sentir dans ses flancs distendus le tressaillement douloureux de l'enfant, tu pourrais te retrouver père d'un Éthiopien ${ }^{52}$. Ici, la femme riche a une sexualité libre, que son mari ne peut restreindre. Ce dernier soutient d'ailleurs son choix de ne pas avoir d'enfant en lui tendant la pharmacopée nécessaire. Juvénal ajoute avec malice que la couleur de la peau de l'enfant révèlerait l'adultère de la femme, acte puni par la loi romaine. D'ailleurs, les témoignages de Cicéron et Plutarque ne sont possibles que dans une société où le refus d'enfanter de la part des femmes est courant. Dans le secret des alcôves, une femme, aidée de sa mère, d'une servante fidèle ou d'une nourrice digne des Hérö̈des, devait pouvoir éviter une naissance qu'elle ne souhaitait pas. Les sources juives révèlent ainsi le cas d'une certaine Judith, vers 330, qui choisit de prendre une potion stérilisante en cachette de son mari : son époux s'en rend compte trop tard ${ }^{53}$. La limitation des naissances est toujours d'actualité dans l'Antiquité tardive : Augustin d'Hippone condamne vers 420 ceux qui refusent d'avoir des enfants ${ }^{54}$, et Césaire d'Arles, au début du VI siècle, blâme les femmes qui après deux ou trois enfants font en sorte d'avorter ou de les tuer $^{55}$.

En ce sens, si les Romains n'hésitaient pas à réguler les naissances, le choix féminin de non-maternité était plutôt secret, le mari étant légalement tout puissant sur le ventre de sa femme. Les philosophes comme le stoïcien Musonius Rufus, qui défendent déjà l'interdiction d'avorter ou d'abandonner ses enfants, sont minoritaires ${ }^{56}$. Le christianisme va radicalement modifier ce rapport à l'enfantement, jugeant toute méthode pour l'éviter immorale ${ }^{57}$. Césaire d'Arles dans un sermon prévient ses paroissiennes : «elles seront tenues pour coupables devant le tribunal du Juge éternel d'autant d'homicides qu'elles auront tué d'enfants déjà conçus ou déjà nés ${ }^{58}$.

\section{Le soutien de l'Église à la non-maternité : des arguments reflet des préoccupations féminines mais à finalité religieuse}

Le christianisme n'oblige pas les femmes à enfanter. L'enseignement chrétien des premiers siècles contient une forte dimension apocalyptique : la fin des temps est proche, et avoir une famille détourne ses pensées de Dieu ${ }^{59}$. Les penseurs chrétiens défendent donc la conversion à la virginité pour les jeunes femmes, à la continence

52 JuvÉNAL, Satires, VI, 592-600, op. cit., traduction légèrement modifiée.

53 Yebamoth, 65 b, Talmud de Babylone.

${ }^{54}$ Augustin D'Hippone, Du mariage et de la concupiscence, V, 5, Paris, Desclée de Brouwer, 1974.

${ }^{55}$ Césaire d’Arles, 52, 4, Sermons, t. 2, Paris, Le Cerf, 1978.

${ }^{56}$ Musonius Rufus, XV A. et XV B., in Entretiens et fragments, traduit par Amand Jagu, New York, Olms Verlag, 1979, p. 71-73.

${ }^{57}$ Et ce dès Tertullien à la fin du $\mathrm{II}^{\mathrm{e}}$ siècle, $c f$. Apologétique, IX, 8, Paris et Louvain, Ch. Peeters, 1911.

58 Césaire d’Arles, 51, 4, Sermons, t. 1, Paris, Le Cerf, 1971.

59 Augustin D’Hippone, «Le bien du mariage», 13, 15, in Problèmes moraux, Paris, 1948 ( $2^{\mathrm{e}}$ ed.), B.A. 2 p. $101-233$ 
pour les époux ${ }^{60}$ et à la chasteté pour les veuves ${ }^{61}$. Le refus de la maternité doit donc s'accompagner de l'abstinence sexuelle, comme l'affirme sans concession Césaire d'Arles : "que la chasteté soit la seule cause de stérilité chez une femme» ${ }^{62}$. Les idées chrétiennes se diffusent dans la société romaine entre le $\mathrm{III}^{\mathrm{e}}$ et le $\mathrm{VI}^{\mathrm{e}}$ siècle, mais les croyances polythéistes persistent tout au long de la période, ce qui nécessite de nuancer leur influence. De plus, la diffusion du christianisme a aussi ses limites : parmi les convertis, beaucoup sont des chrétiens tièdes, absolument pas prêts à renoncer à certaines pratiques traditionnelles.

Les écrivains ecclésiastiques insistent sur les inconvénients de la maternité dans les nombreux traités de virginité ou de chasteté qu'ils composent ${ }^{63}$. La majorité d'entre eux s'essaye au genre, à partir de Tertullien au début du III siècle. Cyprien de Carthage, Ambroise de Milan, Jérôme de Stridon, Augustin d'Hippone, Grégoire de Nysse, Jean Chrysostome veulent convaincre les femmes de ne pas se marier ou de ne pas se remarier. Leurs arguments concernant la maternité sont le reflet des préoccupations des femmes de l'époque. Touchées par ces discours, elles doivent refuser une telle vie pour elles ou pour leurs filles.

En premier lieu, les douleurs et souffrances des femmes enceintes ainsi que celles de l'accouchement sont mises en évidence. Cyprien de Carthage, dès le milieu du $\mathrm{III}^{\mathrm{e}}$ siècle, met en valeur la chance des vierges qui échappent à l'enfantement et ses douleurs ${ }^{64}$. Grégoire de Nysse dans la partie orientale de l'Empire est encore plus explicite dans la seconde moitié du IV siècle : «voici qu'arrive pour la jeune femme l'heure critique de l'enfantement : on entrevoit dans cet évènement non la naissance d'un enfant mais l'arrivée de la mort, et l'on redoute que la parturiente ne meure dans l'accouchement» ${ }^{65}$; ce à quoi fait aussi écho Jean Chrysostome ${ }^{66}$. L'évocation est brutale, mais réaliste : la mortalité des femmes en couches est importante, 5\% à $10 \%$ des femmes ne survivent pas à l'accouchement ou à ses suites, si l'on se fonde sur les chiffres de l'époque moderne. En effet, les séquelles de l'accouchement sont souvent peu ou pas soignées ${ }^{67}$. L'exemple de Mélanie la Jeune, riche héritière

${ }^{60}$ Augustin D’Hippone, «Le bien du mariage», VI, 6, op. cit. Il développe l'idée que la continence doit être choisie d'un commun accord par les époux et non pas unilatéralement. En réalité, c'est surtout le choix de l'époux qui est pris en compte. Dans les récits et correspondances, quand un homme décide de mener une vie chaste, la nécessité de convaincre sa femme n'est jamais mentionnée, tandis que lorsque c'est une femme, elle doit le convaincre, parfois sans succès.

${ }^{61}$ Tertullien, A son épouse, Paris, Le Cerf, 1980, SC n 273. Dans cet ouvrage du tournant $\mathrm{du} \mathrm{III}^{\mathrm{e}}$ siècle, le penseur chrétien Tertullien déconseille à sa femme de se remarier s'il meurt avant elle, thème qui connaît une grande postérité par la suite. Voir aussi JérôME, Lettre 54 à la veuve Furia à laquelle il déconseille de se remarier, Lettres, texte établi et traduit par J. Labourt, t. 3, Paris, Les Belles Lettres, 1953.

${ }^{62}$ Césaire d'Arles, 1, 12, Sermons, t. 2, op. cit.

${ }^{63}$ A. Rousselle, Porneia, op. cit., p. 170-173.

${ }^{64}$ Cyprien, De habitu uirginum, CSEL, 3, 1, op. cit., p. 187-205.

${ }^{65}$ Grégolre de Nysse, Traité de la Virginité, III, 5, Paris, Le Cerf, 1966.

${ }^{66}$ Jean Chrysostome, La Virginité, LVII, 4, Paris, Le Cerf, 1966.

${ }^{67}$ A. Rousselle, «La Politique des corps, entre procréation et continence à Rome», in P. Schmitt Pantel (dir.), Histoire des femmes, t. 1, Paris, Plon, 1991, p. 322. 
romaine convertie à l'ascétisme et à la pauvreté volontaire, qui dirige un monastère de femmes à Jérusalem dans les années 430, permet d'illustrer ces difficultés. Un miracle de Mélanie témoigne plus de connaissances médicales poussées que d'une intervention divine. «Une femme avait eu un accouchement fort difficile, et le fœtus étant mort dans le sein maternel, la malheureuse ne pouvait ni vivre ni trépasser. Ce qu'apprenant, l'authentique servante du Seigneur, compatissante et fort affligée, pleine de pitié pour la femme, de dire aux vierges qui étaient avec elle : "Allons visiter cette femme en danger, afin de voir les souffrances des personnes vivant dans le monde et de comprendre au moins par-là de combien de misère Dieu nous a délivrées" $\gg{ }^{68}$. Mélanie, par la prière, parvient à sauver la femme. L'auteur, Gérontius, sélectionne un miracle qui a plus de chance d'interpeller un lecteur pour qui ce type de drame est courant. Le recours à la prière met en évidence l'impuissance des médecins. De plus, Mélanie a elle-même «eu un accouchement extrêmement difficile» et son fils, né avant terme, est mort juste après sa naissance. La répétition montre bien la fréquence de telles difficultés. Le discours tenu aux vierges par les femmes plus âgées qui les dirigent est lui aussi révélateur de cet état d'esprit. Mélanie leur enseigne non seulement la compassion, mais aussi la conscience de leur privilège de femmes sans sexualité et donc sans maternité.

À ces craintes pour sa propre vie s'ajoutent celles liées à l'enfant. Grégoire de Nysse évalue le drame des jeunes parents, souvent confrontés à la maladie, voire à la mort de leur enfant ${ }^{69}$ comme Jean Chrysostome $^{70}$. La fille de Mélanie et Pinien meurt dans son enfance ${ }^{71}$. La mortalité infantile dans $1^{1} E m p i r e$ romain a été évaluée à environ un enfant sur cinq à partir des inscriptions ${ }^{72}$, même si Rawson propose le chiffre de $50 \%$ de mortalité pour les moins de cinq ans ${ }^{73}$ : une mère de famille risquait donc de voir mourir un ou plusieurs de ses enfants. De surcroît, les appréhensions de la mère doivent aussi être morales quant au devenir de son enfant ${ }^{74}$. Grégoire de Nysse souligne l'incohérence du destin : « celui-ci a en effet perdu le fils de son cœur, celui-là voit survivre le fils libertin; l'un et l'autre sont dignes de pitié, en ce qu'ils déplorent celui-là la mort de son fils et celui-ci sa vie ${ }^{75}$, thème repris par Jean Chrysostome ${ }^{76}$. En ce sens, refuser la maternité revient à refuser beaucoup de turpitudes.

À ces arguments s'ajoutent ceux sur les tracas du mariage et les désagréments d'un époux avec lequel la femme ne vit pas toujours en bonne entente. Donnée très jeune en mariage, sans réel consentement à l'engagement car le père choisit le conjoint ${ }^{77}$,

${ }^{68}$ Gérontius, Vie de sainte Mélanie, IV, 60, Paris, Le Cerf, 1962.

${ }^{69}$ Grégoire de Nysse, Traité de la Virginité, III, 6, op. cit.

70 Jean Chrysostome, La Virginité, LVI, 1, op. cit.

${ }^{71}$ Gérontius, Vie de sainte Mélanie, I, 6, op. cit.

72 A. Rousselle, «La Politique des corps, entre procréation et continence à Rome», loc. cit., p. 321.

${ }^{73}$ B. Rawson, Children and Childhood in Roman Italy, op. cit., p. 341.

${ }^{74}$ CÉsaire D'Arles, Sermons, 51, 3-4, t.2, op. cit.

75 GréGoIre de NysSe, Traité de la Virginité, III, 10, op. cit.

76 Jean Chrysostome, La Virginité, LVII, 5. op. cit.

77 Code Théodosien III, 5, 12, op. cit. 
une femme pouvait vivre son mariage comme un traumatisme, et l'union conjugale comme un viol. Rejet du mariage ou du remariage, et par conséquent du sexe et de la procréation, constituent aussi pour les femmes un choix de non-maternité. Le mariage de la riche Olympias au $\mathrm{IV}^{\mathrm{e}}$ siècle semble bien ne pas avoir été une union heureuse ${ }^{78}$ ce qui a pu l'encourager à rester veuve.

De tels discours ne pouvaient convaincre toute la population, dont une part conséquente restait païenne. Les parents et maris, même chrétiens, s'opposaient régulièrement à de tels choix d'abstinence, souhaitant une descendance ${ }^{79}$. Mélanie ne parvient pas à convaincre son époux Pinien de rester continent, car il veut au moins deux enfants ${ }^{80}$. Pourtant, la répétition de ces arguments avait du sens : une partie des femmes pouvait s'approprier de tels discours, et en user pour justifier leur choix de la virginité ou de la chasteté. Ils servaient aussi à convaincre d'autres femmes d'adopter la même attitude. L'abstinence sexuelle, l'absence de grossesse et d'accouchement sont désormais des garanties de bonne santé dans l'esprit des penseurs chrétiens ${ }^{81}$.

Ce choix est un choix radical. Dans l'esprit des penseurs chrétiens, il doit s'accompagner de l'abstinence sexuelle totale. Les femmes sans maternité doivent être des femmes sans sexualité. Les femmes qui font ce choix dans le contexte chrétien entrent dans un système qui veut régir l'ensemble de leur existence. Les traités, s'ils mettent en avant les bienfaits d'échapper à la maternité, diffusent avant tout un ensemble de règles de vie. La première règle du monastère fondé par Mélanie est que les femmes qui s'y trouvent ne doivent jamais parler avec un homme ${ }^{82}$. Mais beaucoup de femmes continentes ou vierges vivent chez elles ou dans leur famille, où de telles règles ne peuvent pas s'appliquer. Augustin l'affirme : «on ne détestera jamais trop celle qui renonçant au mariage, c'est-à-dire une chose permise, ne renonce pas au péché, soit de luxure, soit d'orgueil, soit de curiosité et de bavardage ${ }^{83}$. Ainsi, si les femmes peuvent choisir - choix conditionné bien souvent à l'obtention de l'accord familial, la volonté familiale à cet égard primant généralement sur les aspirations de la femme ${ }^{84}$, , elles acceptent aussi que les clercs portent sur leur existence un regard sévère. Une coercition morale n'est pas rare, pouvant même devenir physique dans le cadre des monastères ${ }^{85}$. Mais des femmes qui ne sont absolument pas chrétiennes, ou le sont avec un engagement très modéré, peuvent bénéficier des nouvelles possibilités sociales ouvertes par le christianisme sans se plier aux règles que celui-ci prétend imposer.

${ }^{78}$ Vie d'Olympias, III, op. cit.

79 Gérontius, Vie de sainte Mélanie, I, 6, op. cit.

${ }^{80}$ Gérontius, Vie de sainte Mélanie, I, 1, 3, op. cit.

81 J. M. Bennett et R. Mazo Karras, op. cit., p. 87.

${ }^{82}$ Gérontius, Vie de sainte Mélanie, III, 41, op. cit.

${ }^{83}$ Augustin d'Hippone, Le bien du mariage, XII, 14, op. cit.

${ }^{84}$ V. Vuolanto, op. cit., p. 100-122.

${ }^{85}$ Rappelons à la fin du $\mathrm{VI}^{\mathrm{e}}$ siècle la très violente révolte des princesses Basina et Chrodieldis, enfermées contre leur gré au monastère Sainte-Croix de Poitiers et qui fomentent une révolte contre leur abbesse, jugeant que celle-ci leur impose des conditions de vie trop difficiles : faim, dénuement et mauvais traitements. Voir Grégoire DE Tours, Histoire des Francs, V, 39; IX, 34-43 et X, 15-20 notamment. 


\section{Le choix de la non-maternité : un choix pour soi}

En effet, la répétition par les auteurs chrétiens de ces règles de vie où la chasteté est centrale doit poser question. Leur insistance montre que le respect des normes qu'ils cherchent à instaurer est loin d'être effectif. Le christianisme offre un cadre où les femmes peuvent choisir de ne pas avoir d'enfant, mais que leur choix soit lié à des préoccupations religieuses est une autre affaire.

\section{Un choix féminin face aux aléas de l'existence}

Certaines femmes choisissent de ne pas avoir d'enfant car elles ne souhaitent pas avoir un enfant dans la situation qui est la leur, et ce sans distinction de classe sociale.

Nous pouvons évoquer en premier lieu un scandale romain du $\mathrm{II}^{\mathrm{e}}$ siècle touchant des femmes de haut rang. L'évêque Callixte, à Rome, autorisa des femmes chrétiennes d'un certain rang à vivre avec un concubin, libre ou esclave. A. Rousselle les considère comme des filles de sénateurs, qui ne peuvent élever leur mari au rang sénatorial et perdent ce rang en se mariant hors de leur classe ${ }^{86}$. Cette union de circonstances avait sans doute été acceptée par Callixte pour répondre à un déséquilibre entre rang et sexe dans la communauté chrétienne où les femmes de haut rang étaient plus nombreuses que les hommes de même niveau social. Si Hippolyte, rival de Callixte pour la direction de la communauté romaine, critique cette autorisation, il est encore plus outré par ses conséquences. En effet, ces femmes utilisent divers moyens contraceptifs et avortent, ce qui est pour lui contraire à la morale chrétienne. Un tel concubinage ne devait pas engendrer d'enfants, car cette situation devait rester secrète.

Nous sommes ici face à un choix de non-maternité. En effet, elles pourraient très bien se marier dans leur milieu social à un païen et avoir des enfants, ou choisir de rester chastes, ou encore renoncer à leur position sociale et se marier avec un homme d'un rang inférieur. Cette dernière hypothèse aurait pu être mortifère pour la communauté chrétienne : le scandale de telles unions aurait mis les chrétiens dans une position difficile vis-à-vis du pouvoir romain. Ces femmes, d'une réelle éducation, ne pouvaient ignorer que l'oubli de leur rang risquait d'entraîner des persécutions religieuses, pour elles-mêmes, voire pour l'ensemble de leur communauté. Elles choisissent donc de ne pas se priver d'une union, mais sécurisent aussi leur position seul motif égoïste que leur accorde Hippolyte ${ }^{87}$ - ainsi que celle de leur communauté religieuse.

La précarité du statut est aussi un obstacle. Esclaves, captives, concubines peuvent ne pas souhaiter avoir d'enfants tant que leur situation ne s'est pas améliorée. La pauvreté est un motif pour refuser d'avoir des enfants. On peut en premier lieu envisager le cas des concubines, qui peuvent être libres comme esclaves, cas assez bien connu grâce à la législation qui encadre leur statut et celui de leurs enfants, notamment en matière d'héritage. La loi est extrêmement dure envers les concubines et leurs enfants, exclus de la famille légitime. En 336, une loi prohibe ainsi tout droit à un héritage pour les concubines et leurs enfants, et si elles ont reçu quoi que ce soit avant la mort de l'homme avec lequel elles vivaient en concubinage, elles doivent le

\footnotetext{
86 A. Rousselle, Porneia, op.cit., p. 136-137.

87 Hippolyte de Rome, Philosophumena, op. cit.
} 
rendre ${ }^{88}$. Une loi de $371^{89}$ redonne malgré tout un peu d'espoir aux concubines : par héritage ou par donation, un douzième des biens peut désormais aller à une femme quelconque, ou aux enfants issus d'une telle union. Et s'il n'y a pas d'enfants ni de petits-enfants d'un mariage légitime, c'est un quart de ses biens que l'homme peut alors donner, loi confirmée en $405^{90}$. Mais la réalité reste malgré tout rude pour ces femmes : le mariage est un arrangement pour obtenir une progéniture légitime, et conserver un patrimoine. Par conséquent, les concubines, sans réseau ni soutien familial, devaient facilement être exclues. La précarité de ces dernières peut leur faire souhaiter de ne pas avoir d'enfant. Outre les risques pour leur santé, l'avenir de leur progéniture n'est pas assuré, et certains hommes ne souhaitent pas non plus que leur concubine soit mère : elles risquent donc de perdre leur soutien financier. Bien évidemment, les femmes libres engagées dans une relation de concubinage ont une capacité de choix plus grande que les esclaves. Mais si on songe au vieillissement précoce engendré par des grossesses répétées et aux complications médicales qui les suivent, sans compter le risque de mortalité en couches, elles devaient fréquemment avorter.

L'avortement et l'infanticide peuvent être pour une esclave des moyens de résistance à l'esclavage, d'autant plus qu'à Rome la servilité est héréditaire. Ce refus est un choix dangereux pour une esclave : comme l'enfant à naître est la propriété du maître, c'est une atteinte à ses revenus que d'avorter ou de le tuer à la naissance. Selon Noonan ${ }^{91}$, ce choix se faisait surtout dans les contextes où la vie de l'esclave était vraiment difficile, avec de fortes disparités selon les lieux et les époques. A. Rousselle cite en exemple le canon 5 du Concile d'Elvire, qui prive de communion pendant sept années la femme qui aurait fouetté sa servante si fort que celle-ci en serait morte dans les trois jours ${ }^{92}$. Dans la même optique, Césaire d'Arles parle à ses fidèles : «Je voudrais bien savoir si une femme de condition libre qui absorbe des drogues porteuses de mort pour ne pas concevoir voudrait que ses servantes ou ses fermières en fissent autant. Et c'est pourquoi, de même que chacune veut que lui naissent des esclaves pour la servir, qu'elle nourrisse aussi elle-même tous les enfants qu'elle a conçus ou bien qu'elle confie à d'autres le soin de les nourrir, qu'elle ne refuse pas de les concevoir ou, ce qui est plus grave, qu'elle ne désire pas tuer ceux qui auraient pu être de bons chrétiens $»{ }^{93}$. Césaire veut ainsi mettre en évidence l'incohérence des riches propriétaires qui veulent choisir pour elles-mêmes, mais pour des raisons économiques, ne veulent pas laisser ce choix à leurs servantes. Les pratiques de la période précédente, contraception, avortement, exposition des enfants

88 Code Théodosien IV, 6, 3, op. cit.

${ }^{89}$ Code Théodosien IV, 6, 4, op. cit.

90 Code Théodosien IV, 6, 6, op. cit.

91 J. T. NoOnan, op. cit. p. 31-32.

92 A. Rousselle, «La Politique des corps, entre procréation et continence à Rome», loc. cit., p. 357.

93 Césaire D’Arles, Sermons, t. 2, 44, 2, op. cit. 
continuent de se maintenir ${ }^{94}$. À l'opposé, la femme esclave pouvait être aussi forcée à l'abstinence, si ses maîtres considéraient l'abstinence en plus haute estime ${ }^{95}$.

Aux questions de rang social s'ajoute celle du statut religieux. Les vierges consacrées et veuves enregistrées auprès d'une communauté ecclésiastique, si elles sont d'un niveau social élevé, ont une réputation à perdre, et si elles sont pauvres, le soutien financier de l'Église à conserver. Celles-ci vont vouloir à tout prix éviter d'avoir des enfants. Tertullien dénonce ainsi les vierges qui veulent dissimuler leur forfait : «Que n'ira-t-elle aussi essayer sur son ventre, afin de cacher sa grossesse ?» ${ }^{96}$ Le témoignage d'Ambroise, près de deux siècles plus tard, à propos de la vierge Indicia accusée d'avoir mis un enfant au monde, et de l'avoir exposé ou tué, montre bien le caractère scandaleux de ce genre d'évènements ${ }^{97}$.

De même, dans certains métiers féminins, la maternité n'est pas souhaitable. Il n'est pas souhaitable que les prostituées aient des enfants, idée que l'on trouve chez Lucrèce au I ${ }^{\text {er }}$ siècle ${ }^{98}$. Augustin d'Hippone, au IV ${ }^{\mathrm{e}}$ siècle, reprend la même idée à propos des manichéens, mouvement qu'il dénonce comme hérétique. Ceux-ci ne voulant pas d'enfants, chacun «fait de la femme non plus une épouse, mais une prostituée $»{ }^{99}$. Jean Chrysostome s'inquiète que de nombreuses épouses se comportent comme des prostituées ${ }^{100}$. Il y a stigmatisation de la prostitution, et mise en évidence que les prostituées sont les premières à refuser la maternité. D'autres professions pouvaient aussi être durement impactées par une grossesse, comme les chanteuses ${ }^{101}$, comédiennes ou danseuses. Libres, elles attendaient le bon moment ou refusaient la précarité dans laquelle une grossesse les porterait, esclaves, elles pouvaient aussi refuser ce choix d'enfanter s'il n'était pas fait à leur place et contre leur gré.

\section{Un choix par rapport à des aspirations personnelles}

À ces motivations très concrètes s'ajoutent d'autres motifs. De nombreuses femmes semblent faire le choix chrétien de non-maternité, et respectent les règles définies par les clercs. Mélanie a dès l'enfance une vocation à une vie de chasteté ${ }^{102}$, comme Scolastica, la sœur de Benoît de Nursie ${ }^{103}$. Pour Olympias, la perte brutale de son mari après quelques mois de mariage lui laisse penser que Dieu veut qu'elle

${ }^{94}$ K. HARPER, Slavery in the Late roman world, New York, Cambridge University Press, 2011, p. 393.

${ }^{95}$ Ibid., p. 295-298.

96 Tertullien, Le Voile des vierges, 14, 7, Paris, Le Cerf, 1997.

${ }^{97}$ Ambroise, Lettre 5, et en particulier 5,12, PL 16, 929-937. Pour une traduction récente, en anglais, M. Beyenka, Ambrose's Letters, 1-91, Fathers of the Church 26, Washington D.C., Catholic University Press, 2002, épître 32.

98 LUCRĖCE, De la nature, IV, 1268-1277, op. cit.

${ }^{99}$ Augustin D'Hippone, Les Mours des Manichéens, XVIII, 65, introduction, traduction et notes par B. Roland-Gosselin, Paris, Desclée, Brouwer et $\mathrm{C}^{\text {ie }}$ (Bibliothèque augustinienne $\left.\mathrm{n}^{\circ} 1\right), 1949$.

100 Jean Chrysostome, Homélie sur l'épître aux Romains, 24, 3, Bar-le-Duc, L. Guérin \& Cie, 1864.

${ }^{101}$ S. Gourevitch, op. cit. p. 275.

102 Gérontius, Vie de Sainte Mélanie, I, 1. op. cit.

${ }^{103}$ Grégoire le Grand, Dialogues, II, 33, 2-4, Paris, Le Cerf, 1979. 
soit continente et qu'elle ne se remarie pas ${ }^{104}$. Au-delà de la rhétorique chrétienne convenue, ces femmes pouvaient trouver de véritables attraits à un choix ascétique. En effet, ne pas se marier et ne pas avoir d'enfants signifiait une vie nettement plus libre : possibilité de voyager, contrôle sur leur emploi du temps et leur fortune, possibilité d'échanges intellectuels et de se consacrer à l'étude ; mais ces arguments semblent surtout valables pour les femmes de l'élite. On les retrouvera au XII ${ }^{\mathrm{e}}$ siècle dans la bouche d'Héloïse, jugeant aussi la maternité incompatible avec l'étude des Écritures ${ }^{105}$.

Malgré tout, un certain nombre de femmes semble bien négliger tant les conventions sociales que les recommandations des penseurs chrétiens dans leur choix de non-maternité. En effet, certaines femmes qui choisissent de ne pas avoir d'enfants n'ont aucunement l'intention de se priver des plaisirs de l'existence pour mener une vie d'ascétisme chrétien. Les auteurs chrétiens insistent tout particulièrement sur tout ce que les femmes célibataires ne doivent pas faire : «Quant à la virginité, elle peut exister sans l'obéissance, parce qu'une femme qui a pris la résolution d'être vierge et s'y tient fidèlement peut transgresser les commandements de Dieu. Nous connaissons en effet beaucoup de personnes consacrées à Dieu qui sont bavardes, curieuses, adonnées au vin, brouillonnes, avares, orgueilleuses $\gg{ }^{106}$. Augustin souligne que si certaines font le choix de ne pas avoir d'enfants, ce n'est pas pour servir le dieu chrétien, mais pour vivre sans entraves. Cyprien de Carthage, un siècle et demi auparavant, ne disait pas autre chose, critiquant les vierges qui vont danser aux noces et entendent des choses inconvenantes, sans compter celles qui se mêlent aux hommes dans les bains publics sans y voir à mal ${ }^{107}$. Pour Jérôme, «la virginité peut se perdre aussi par la simple pensée » ${ }^{108}$. Il donne d'ailleurs la parole aux femmes qui bien que respectant la chasteté, sont enclines à profiter des plaisirs de la vie : «Ce sont celles-là qui ont coutume de dire : “tout est pur pour les purs! Ma conscience me suffit. C'est un cœur pur que désire Dieu, pourquoi me priver de nourritures que Dieu a créées pour qu'on en use?" Si elles se mettent en frais de charme et de gaieté, elles se gorgent de vin pur, puis joignant à l'ébriété le sacrilège, elles s'exclament : "Bien sûr que non, je ne m'abstiendrai pas du sang du Christ!" $»{ }^{109}$. La violence hiéronymienne ne doit pas masquer la cohérence du discours de ces femmes. Respecter la chasteté, soit, mais elles ne voient pas pourquoi elles devraient se priver des plaisirs du monde.

Certaines semblent au contraire, toujours selon Jérôme, avoir honte de leurs pratiques ${ }^{110}$. Elles se donnent une apparence austère sans en avoir le comportement. Tout chez elles est jeu d'actrice, considérant déjà que l'habit fait le moine : «Aperçoiventelles quelqu'un? Aussitôt elles gémissent, abaissent les paupières, se couvrent la figure; c'est tout juste si elles libèrent un œil pour regarder. La robe est grossière, la

${ }^{104}$ Vie d'Olympias, III, Paris, Le Cerf, 1968.

105 C. Leyser et L. Smith, « Preface », in Motherhood, Religion and Society in Medieval Europe, 400-1400, op. cit., p. xiii.

106 Augustin D’Hippone, Le bien du mariage, XXIII, 30, op cit.

107 Cyprien, De Habitu virginum, 5, op cit.

108 JÉrôme, Lettres, 22, 5 à Eustochium, t. 1, Paris, Les Belles Lettres, 1949, p. 110-160.

109 JÉrôME, Lettres, 22, 13, op. cit.

110 JÉRÔME, Lettres, 22, 27, op. cit. 
ceinture de vil tissu, les mains et les pieds sales; mais l'estomac, lui, parce qu'on ne peut le voir, étouffe de mangeaille ». Les femmes connaissent les attentes sociales. La femme qui échappe à son rôle de mère pourrait être regardée avec désapprobation, si elle profite de sa liberté au lieu de mortifier son corps. Ces femmes sans enfant et sans vocation religieuse, ou avec une interprétation des textes qui leur est propre vivent heureuses, mais cachées. Leur choix de non-maternité est un choix personnel, qu'elles ne peuvent déclarer ouvertement au reste de la société, au risque de faire scandale. Mais elles le vivent malgré tout, et si Jérôme a pu percevoir le mensonge au-delà de leur apparence ascétique, c'est bien que leur mode de vie est souvent un secret de polichinelle. Tant qu'elles restent discrètes, la société accorde une certaine tolérance à leurs pratiques. Qu'elles dissimulent, c'est déjà admettre aux yeux de la morale publique qu'elles ont tort, et leur culpabilité affichée par leur comportement de tragédienne suffit à maintenir l'illusion de la morale publique. L'exemple d'Indicia, vierge consacrée qui aurait été enceinte et aurait tué son enfant à sa naissance illustre bien comment l'ensemble d'une communauté peut s'impliquer dans ce type de débat moral, des servantes aux voisins et à l'évêque ${ }^{111}$.

Plus encore, si celles-ci peuvent encore respecter la règle chrétienne de la chasteté comme seule stérilité, d'autres n'ont même pas cette inquiétude. Les pratiques de contraception et d'avortement semblent courantes chez les femmes veuves ou mariées, elles «dégustent d'avance les commodités de la stérilité; elles tuent un être humain avant qu'il ne soit procréé. Plusieurs, quand elles s'aperçoivent qu'elles ont conçu dans le crime, songent aux poisons qui font avorter. Souvent elles en meurent aussi de même coup ${ }^{112}$.

Ce choix de non-maternité peut également être encouragé par certains mouvements chrétiens concurrents du christianisme nicéen et déclarés hérétiques. Les manichéens considèrent ainsi que le mariage existe pour assouvir ses désirs charnels et non pour avoir des enfants ${ }^{113}$. De telles prescriptions existent aussi chez les priscillianistes, comme l'écrit en 447 le pape Léon Ir $^{\text {e }}$ dans sa lettre au nouvel évêque d'Astorga en Espagne : ceux-ci refusent le mariage et la procréation, mais acceptent les relations sexuelles. Malgré l'exécution de leur meneur et leur persécution, ils ont profité des guerres incessantes pour diffuser leurs théories, qui rencontrent un certain succès en Espagne ${ }^{114}$.

Un cas particulier de non-maternité à signaler est celui des mères qui ne se préoccupent pas de leurs enfants déjà nés, et les confient à des proches ou à un monastère plutôt que de les élever ${ }^{115}$. Dans les sources ecclésiastiques, cette entrée au monastère est présentée comme un souhait de l'enfant lui-même ou comme le résultat

${ }_{111}$ Ambroise, Lettres, 5, op. cit.

112 JÉrôMe, Lettres, 22, 13, op. cit.

113 Augustin d'Hippone, Les Mours des Manichéens, 18, 65, op. cit.

114 LÉON, XV, 1 et 7, Lettres, Patrologia Latina 54, Paris, J.-P. Migne, 1846, p. 679 et 683. Pour une traduction française, voir E. de Montferrier, Chefs-d'œuvre des Pères de l'Église, t. $14,1838$.

${ }^{115}$ Cf. V. Vuolanto, op. cit., p. 102-104. Fl. Jullien, «L'accueil des enfants en milieu monastique syriaque $\left(\mathrm{V}^{\mathrm{e}}-\mathrm{VIII}{ }^{\mathrm{e}}\right.$ siècles)», carnet Hypothèses : HospitAm en ligne : https:// hospitam.hypotheses.org/729, dernière consultation le 17 mai 2018. 
d'un vœu de ses parents. Mais il faut envisager le cas d'enfants abandonnés par leurs parents et recueillis par de riches ascètes, par des prêtres ou par des monastères ${ }^{116}$. Les chrétiens abandonnent leurs enfants ${ }^{117}$. Cela semble être le cas de Gérontius, recueilli par Mélanie et Pinien, qui leur en est très reconnaissant, mais reste silencieux sur sa situation antérieure. La seule véritable mention d'une mère abandonnant totalement son enfant à un proche existe, dans la correspondance d'Ennode de Pavie au VI ${ }^{\mathrm{e}}$ siècle. Sa sœur veuve Euprepia lui a confié son fils unique Lupicinus pour qu'il l'éduque, sans jamais lui donner de nouvelles, ce qu'il lui reproche dans ses lettres ${ }^{118}$. Mère malgré elle, Euprepia ferait un choix de non-maternité bien après la naissance de son enfant, choix qu'elle n'aurait pas pu faire auparavant, du vivant de son mari.

\section{Conclusion}

Réfléchir sur le choix de la non-maternité dans l'Antiquité tardive permet de mettre en évidence diverses évolutions. D'une maternité encouragée par la loi et d'un célibat pénalisé depuis Auguste, on passe au IV siècle à un célibat librement choisi sans pénalités légales, auquel va même s'ajouter un arsenal législatif protégeant les vierges et les veuves. La fiabilité des moyens de contraception ou d'avortement reste toujours discutable, même si ces pratiques semblent répandues. Le problème majeur qui se pose alors est celui d'un choix qui n'est que rarement celui de la femme. Le choix de l'époux reste déterminant. Les femmes qui choisissent de ne pas avoir d'enfants doivent alors souvent dissimuler leurs pratiques pour l'éviter. Le soutien de l'Église chrétienne à ce choix féminin de non-maternité constitue une nouveauté dans l'Antiquité tardive, même s'il le conditionne à un mode de vie ascétique. Malgré tout, son influence reste limitée, avec une adhésion toujours partielle des populations au christianisme et une foi qui reste modérée chez nombre de convertis. Les textes chrétiens nous permettent de mieux comprendre pourquoi les femmes pouvaient refuser la maternité en mettant en évidence les risques et les souffrances de celle-ci pour la femme, les soucis que l'éducation des enfants entraîne mais aussi les difficultés relationnelles possibles avec un mari la plupart du temps imposé par le père. D'autres raisons concrètes peuvent de surcroît mener les femmes à ce choix de non-maternité. Statut social, métier, préoccupations et aspirations personnelles comptent aussi. Le choix de non-maternité n'a pas que des motivations chrétiennes. Mais toutes les femmes, chrétiennes ou non, peuvent tirer parti de ce nouveau cadre social pour s'épanouir en dehors de la maternité. L'enfermement conventuel, qui forcera les femmes célibataires au respect des règles ascétiques, ne devient la norme

116 J. E. Boswell, « Expositio and Oblatio: The Abandonment of Children and the Ancient and Medieval Family », loc. cit., p. 17-21 met en évidence le recours à cette pratique tout-aulong du Moyen-Âge, notamment dans des situations de pauvreté ou en cas de famine.

117 J. E. Boswell, The Kindness of Strangers. The Abandonment of Children in Western Europe from Late Antiquity to the Renaissance, op. cit., p. 177.

118 Ennode de Pavie, Lettres, III, 15 et 28, t. 2, livres III et IV, Paris, Les Belles Lettres, 2010. 
qu'à l'époque carolingienne, réduisant la capacité d'action des femmes ne désirant pas d'enfants ${ }^{119}$.

\section{Bibliographie}

Aëtios d'Amida, Médecine, Leipzig, S. Zervos, 1901.

Ambroise, Lettre 5, in M. BeyenkA, Ambrose's Letters, Washington D.C., Catholic University Press, 2002.

Ambroise de Milan, Hexaméron, Corpus scriptorum ecclesiasticorum latinorum (CSEL), t. 32.

Augustin D’Hippone, Du mariage et de la concupiscence, Paris, Desclée de Brouwer, 1974.

Augustin D’Hippone, «Le bien du mariage», in Problèmes moraux, Paris, 1948.

Augustin D'Hippone, Les Mours des Manichéens, introduction, traduction et notes par

B. Roland-Gosselin, Paris, Desclée, Brouwer et $\mathrm{c}^{\text {ie }}$ (Bibliothèque augustinienne $\left.\mathrm{n}^{\circ} 1\right), 1949$.

Bennett J. M. et Mazo Karras R., The Oxford handbook of women and gender in Medieval Europe, Oxford, Oxford University Press, 2013.

Berger A., « Encyclopedic Dictionary of Roman Law », Transactions of the American Philosophical Society, n ${ }^{\circ}$ 43.2, 1953.

Boehringer S., L'Homosexualité féminine dans l'Antiquité grecque et romaine, Paris, Les Belles Lettres, 2007.

Boswell J. E., « Expositio and Oblatio: The Abandonment of Children and the Ancient and Medieval Family », The American Historical Review, vol. 89, ${ }^{\circ} 1$, 1984, p. 10-33.

Boswell J. E., The Kindness of Strangers. The Abandonment of Children in Western Europe from Late Antiquity to the Renaissance, New York, Pantheon books, 1988.

BROOTEN E., Love between women, early Christian responses to female homoeroticism, Chicago, University of Chicago Press, 1996.

Brown P., Le Renoncement à la chair, Paris, Gallimard, 1996 [1995].

Césaire D’Arles, Sermons, t. 1, Paris, Le Cerf, 1971.

Césaire D’Arles, Sermons, t. 2, Paris, Le Cerf, 1978.

CicÉron, Discours, tome VIII, Paris, Les Belles Lettres, 1953.

Clément D’Alexandrie, Stromates, III, in Écrits apocryphes chrétiens, Paris, Gallimard (coll. « La Pléiade »), 1997.

Code Théodosien VIII, 16, 1, version latine et traduction française, in P. LAURENCE, Les droits de la femme au Bas-Empire romain : le Code Théodosien, Neuvillesur-Saône, Chemins de traverse, 2012.

Corbier M., " Child Exposure and Abandonment », in S. Dixon (éd.), Childhood, Class and Kin in the Roman World, Londres, Routledge, 2001, p. 52-73.

CYPRIEN, De habitu uirginum, Corpus scriptorum ecclesiasticorum latinorum (CSEL), t. 3 .

119 E. SANTINELli, Des femmes éplorées? Les veuves dans la société aristocratique du haut Moyen Âge, Paris, Presses universitaires du Septentrion, 2003, p. 165-166. 
Digeste, in D. GAURIER, Les 50 livres du Digeste de l'Empereur Justinien : comprenant pour la première fois en français La Palingénésie du commentaire sur l'édit du préteur d'Ulpien (d'après Otto Lenel), Nantes, Mémoires du droit, 2017.

Duby G. et Perrot M., Histoire des femmes en Occident, Paris, Plon, 1990-1991 (5 volumes).

EnNode de Pavie, Lettres, t. 2, Paris, Les Belles Lettres, 2010.

Eyber E., " Family planning in Graeco-Roman Antiquity », Ancient Society, vol 11/12, Louvain, 1980-1981, p. 5-82.

FrIER B. W., « Natural Fertility and Family Limitation in Roman Marriage », Classical Philology, vol. 89, n², 1994, p. 318-333.

Garnsey P., « Child rearing in Ancient Italy », in D. I. Kertzer et R. P. Saller (eds.), The Family in Ancient Italy from Antiquity to the Present, New Haven, Londres, Yale University Press, 1992, p. 48-65.

Gérontius, Vie de sainte Mélanie, Paris, Le Cerf, 1962.

Gourevitch S., Le mal d'être femme. La femme et la médecine dans la Rome antique, Paris, Les Belles Lettres, 1984.

GréGoire de Nysse, Traité de la Virginité, Paris, Le Cerf, 1966.

GréGoire le Grand, Dialogues, Paris, Le Cerf, 1979.

Надот P., «Le Gnosticisme», in Dictionnaire de l'Histoire du christianisme, Paris, Encyclopaedia Universalis, Albin Michel, 2014, p. 641.

HarPer K., Slavery in the Late roman world, New York, Cambridge University Press, 2011.

Harris W. V., " Child-Exposure in the Roman Empire », Journal of Roman Studies, 84, p. 1-22.

Himes N., Medical history of contraception, Baltimore, The Williams and Wilkins Company, 1936.

Hippolyte de Rome, Philosophumena, Paris, Rieder, 1928.

Hopkins K., "Contraception in the Roman Empire », Comparative Studies in Society and History, vol. 8(1), 1965, p. 124-151.

Jean Chrysostome, Homélie sur l'épître aux Romains, Bar-le-Duc, L. Guérin \& Cie, 1864

Jean Chrysostome, La Virginité, Paris, Le Cerf, 1966.

JÉRÔME, Lettres, t. 1, Paris, Les Belles Lettres, 1949.

JÉRÔME, Lettres, t. 3, Paris, Les Belles Lettres, 1953.

JULLIEN Fl., «L'accueil des enfants en milieu monastique syriaque (Ve-VIII ${ }^{\mathrm{e}}$ siècles)», carnet Hypothèses : HospitAm en ligne : https://hospitam.hypotheses.org/729.

JuvÉnAL, Satires, Paris, Les Belles Lettres, 1951.

KNiBeineler Y., Histoire des mères et de la maternité en Occident, Paris, PUF, 2000.

Leyser C. et Smith L., Motherhood, Religion and Society in Medieval Europe, 4001400, Burlington, Farnham, Ashgate, 2011.

LÉon, Lettres, Patrologia Latina, Paris, J.-P. Migne, 1846.

McLaren A., A History of contraceptions from Antiquity to the Present day, Oxford, Blackwell, 1990.

Musonius Rufus, XV A. et XV B., in Entretiens et fragments, traduit par Amand Jagu, New York, Olms Verlag, 1979. 
Noonan J. T., Contraception et mariage, Paris, Le Cerf, 1969 [1966].

Octavius, Paris, Les Belles Lettres, 1964.

Ovide, Héroïdes, Paris, Les Belles Lettres, 1928.

Plutarque, Vies, t. 1, Paris, Les Belles Lettres, 1957.

Riddle J. M., Contraception and Abortion from the Ancient world to Renaissance,

Cambridge (Mass.), Harvard University Press, 1992.

Rousselle A., «La Politique des corps, entre procréation et continence à Rome», in

P. Schmitt Pantel (dir.), Histoire des femmes, t. 1, Paris, Plon, 1991, p. 385-439.

Rousselle A., Porneia, Paris, PUF, 1983.

Rawson B., Children and Childhood in Roman Italy, Oxford, Oxford University Press, 2003.

Santinelli E., Des femmes éplorées? Les veuves dans la société aristocratique du haut Moyen Âge, Paris, Presses universitaires du Septentrion, 2003.

SÉNĖQue le RhÉTeur, Controverses et suasoires, introduction, traduction et notes

d'H. Bornecque, Paris, Garnier, 1932.

Soranos d'Éphèse, Maladies des femmes, t. 1, Paris, Les Belles Lettres, 1988.

TAte J. C., « Christianity and the Legal Status of Abandoned Children in the Later

Roman Empire », Journal of Law and Religion, vol. 24, n¹, 2008/2009, p. 123141.

Tertullien, Apologétique, Paris et Louvain, Ch. Peeters, 1911.

Tertullien, A son épouse, Paris, Le Cerf, 1980.

Tertullien, Le Voile des vierges, Paris, Le Cerf, 1997.

Veyne P., «L'Empire romain », in G. Duby et P. Ariès (dir.), Histoire de la vie privée, t. 1, Paris, Le Seuil, 1985, p. 20-223.

Vuolanto V., Children and Asceticism in Late Antiquity, Burlington, Farnham, Ashgate, 2015.

Yebamoth, 65 b, Talmud de Babylone. 